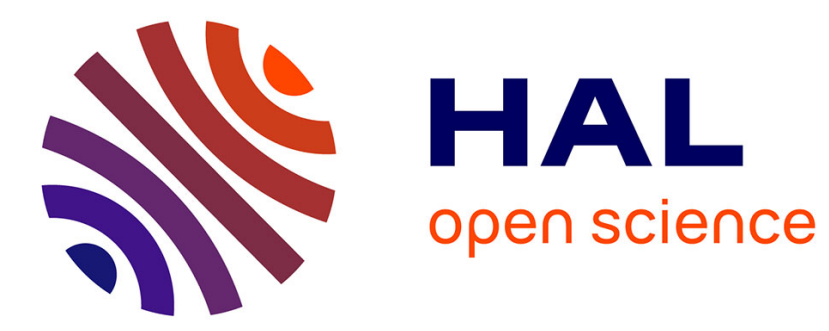

\title{
Spatial Multiplexing applied to Turbo Coded Multi-Carrier CDMA
}

Vincent Le Nir, Maryline Hélard, Rodolphe Le Gouable

\section{To cite this version:}

Vincent Le Nir, Maryline Hélard, Rodolphe Le Gouable. Spatial Multiplexing applied to Turbo Coded Multi-Carrier CDMA. 2004, 5 p. hal-00005808

\section{HAL Id: hal-00005808 \\ https://hal.science/hal-00005808}

Submitted on 4 Jul 2005

HAL is a multi-disciplinary open access archive for the deposit and dissemination of scientific research documents, whether they are published or not. The documents may come from teaching and research institutions in France or abroad, or from public or private research centers.
L'archive ouverte pluridisciplinaire $\mathbf{H A L}$, est destinée au dépôt et à la diffusion de documents scientifiques de niveau recherche, publiés ou non, émanant des établissements d'enseignement et de recherche français ou étrangers, des laboratoires publics ou privés. 


\title{
Spatial Multiplexing applied to Turbo Coded Multi-Carrier CDMA
}

\author{
Vincent Le Nir, Maryline Hélard, Member, IEEE, Rodolphe Le Gouable \\ France Telecom R\&D, 4 rue du Clos Courtel BP 91226 - 35512 Cesson Sévigné Cedex, FRANCE \\ E-mail: vincent.lenir@rd.francetelecom.com
}

\begin{abstract}
In this paper, we combine Spatial Multiplexing with a Multi-Carrier Code Division Multiplex Access (MC-CDMA) system associated to a Turbo Coding (TC) scheme. MC-CDMA is likely to be a very promising access technique for future wireless communication systems. In fact, MC-CDMA exploits the advantages of both multi-carrier modulation and Code Division Multiple Access (CDMA) technique. On the other hand, the capacity of multi-antenna system can linearly increase with the minimum of transmit and receive antennas using Spatial Multiplexing. We study the concatenation of Multiple Input Multiple Output (MIMO) systems for $N_{t}$ transmit and $N_{r}$ receive antennas and MC-CDMA with a TC scheme. Simulation results are provided for different loads in order to demonstrate the efficiency of spatial multiplexing combined with turbo channel coding scheme for a multi-user system based on MC-CDMA for both uncorrelated and correlated antenna systems.
\end{abstract}

\section{INTRODUCTION}

On one hand, MC-CDMA is a very promising technique for the future wireless communication systems. This technique combines Orthogonal Frequency Division Multiplex (OFDM) multi-carrier modulation and CDMA access technique deriving benefits from both techniques increasing the high spectral efficiency and the robustness against multi-path channels. The OFDM modulation consists in splitting the initial data rate in lower data rates transmitted over nonselective frequency subcarriers. Moreover, the multi access flexibility and low multiuser interference are provided by CDMA [1]. On the other hand, multiple antenna systems were also demonstrated to significantly increase the capacity of future wireless communication systems [2]. In this paper, we briefly present a MC-CDMA system in a Single Input Single Output (SISO) antenna system before extending MC-CDMA to a MIMO system with $N_{t}$ transmit and $N_{r}$ receive antennas. In [3][4], it was demonstrated that a good tradeoff between complexity and BER performance is achieved by using a Single User (SU) Minimum Mean Square Error (MMSE) detector with a Turbo Coding (TC) scheme for a SISO MCCDMA transmission. Indeed, it was shown in [3] that more complex Multi User (MU) Successive Interference Canceller
(SIC) or Parallel Interference Canceller (PIC) detectors lead to worse performance results than a SU MMSE one when MC-CDMA is associated to a TC scheme. Moreover, for a MIMO scheme, the Vertical Bell Labs Advanced Space-Time (VBLAST) receiver uses a SIC detector [5] in order to retrieve the information of the multi-antenna transmitted symbols. In this paper, we associate a TC scheme and a SU MMSE detector with the MIMO MC-CDMA system. We compare two types of MIMO receivers which are the SU MMSE detector adapted to spatial multiplexing with or without a multi-antenna SIC detection. We provide simulation results over uncorrelated Rayleigh flat fading channels per V-BLAST for different loads for both uncorrelated or correlated antenna systems.

\section{MC-CDMA, PRESEnTATion}

In this section we briefly present the principle of a MCCDMA system. First, data bit stream of each user is individually turbo coded and converted into a symbol vector. The multiuser turbo coded symbol matrix $\mathbf{X}$ including the information of all the users is of size $N_{u} \times N$ and denoted $\mathbf{X}=\left[\begin{array}{lllll}\mathbf{x}_{1} & \ldots & \mathbf{x}_{n} & \ldots & \mathbf{x}_{N}\end{array}\right]$ where $n$ is the time index and $N$ the number of symbol vectors transmitted during $N$ symbol durations. $\mathbf{x}_{n}=\left[\begin{array}{lllll}x_{1 n} & \ldots & x_{j n} & \ldots & x_{N_{u} n}\end{array}\right]^{T}$ is a vector of length $N_{u}$, where $N_{u}$ is the number of users and [.] $]^{T}$ the transpose operation. The multiuser coded sequence is spread using a Fast Hadamard Transform (FHT). We consider that the length of the spreading sequences is equal to $L_{c}$. Here we assume $L_{c} \leq N_{c}$, where $N_{c}$ is the number of subcarriers of the OFDM. In practice, OFDM modulation and demodulation are easily carried out in the digital domain by performing Inverse Fast Fourier Transform (IFFT) and FFT operations. Furthermore the insertion of a guard interval between adjacent OFDM symbols guarantees the absence of Inter Symbol Interference (ISI). In this paper, uncorrelated frequency nonselective Rayleigh fading per subcarrier is considered. The theoretical channel response of the $k$ th subcarrier can be estimated by $h_{k}=\rho_{k} e^{i \theta_{k}}$ due to perfect orthogonality in synchronous transmission [4]. In the SISO case, the received 
signals for the $L_{c}$ subcarriers can be expressed by:

$$
\mathbf{R}=\mathbf{H C X}+\mathbf{N}
$$

where $\mathbf{R}=\left[\begin{array}{lllll}\mathbf{r}_{1} & \ldots & \mathbf{r}_{n} & \ldots & \mathbf{r}_{N}\end{array}\right]$ is a matrix of size $L_{c} \times N$, with $\mathbf{r}_{n}=\left[\begin{array}{llllll}r_{1 n} & \ldots & r_{k n} & \ldots & r_{L_{c} n}\end{array}\right]^{T}$ the received vector of length $L_{c}$ on the different subcarriers. $\mathbf{H}$ is a $L_{c} \times L_{c}$ time varying diagonal matrix, each element of the diagonal standing for the frequency channel response of each subcarrier, $\mathbf{C}=\left[\begin{array}{lllll}\mathbf{c}_{1} & \ldots & \mathbf{c}_{j} & \ldots & \mathbf{c}_{N_{u}}\end{array}\right]$ is the $L_{c} \times N_{u}$ matrix of user's spreading codes, $\mathbf{c}_{j}=\left[\begin{array}{lllll}c_{1 j} & \ldots & c_{k j} & \ldots & c_{L_{c} j}\end{array}\right]^{T}$ is the vector of length $L_{c}$ of user $j$, and $\mathbf{N}$ is the Additive White Gaussian Noise (AWGN) matrix of size $L_{c} \times N$. At the receiver side, the orthogonality between users has to be restored by applying an equalization process before despreading. In this paper, we only consider a SU MMSE detector. In fact, for a SISO MC-CDMA transmission with TC, a SU MMSE detector was demonstrated to be a good tradeoff between complexity and performance, avoiding the use of more complex MU detectors like SIC or PIC detectors [3].

Hence, at the receiver in case of SU detector, an equalizer $g_{k}$ corrects the $L_{c}$ amplitude and phase variations of each subcarrier $k . \mathbf{G}$ is a diagonal matrix containing the equalization coefficients $g_{k}$. We can consider that

$$
\mathbf{G}=\frac{\mathbf{H}^{H}}{\mathbf{H}^{H} \mathbf{H}+\frac{1}{\gamma} \mathbf{I}}
$$

corresponding to the MMSE SU equalization matrix where $[.]^{H}$ denotes the transpose conjugate and $\gamma$ the input Signal to Noise Ratio (SNR) at the receive antenna. Thus, the equalization coefficient can be written as follows:

$$
g_{k}=\frac{h_{k}^{*}}{\left|h_{k}\right|^{2}+\frac{1}{\gamma}}
$$

After equalization, the resulting signal is:

$$
\mathbf{Y}=\mathbf{G H C X}+\mathbf{G N}
$$

where $\mathbf{Y}=\left[\mathbf{y}_{1} \ldots \mathbf{y}_{n} \ldots \mathbf{y}_{N}\right]$ is the $L_{c} \times N$ matrix of the equalized signals, with $\mathbf{y}_{n}=\left[\begin{array}{lllll}y_{1 n} & \ldots & y_{k n} & \ldots & y_{L_{c} n}\end{array}\right]^{T}$ the $L_{c}$ length vector of the received symbol $n$. The final step consists in executing the despreading by applying the IFHT to the vector $\mathbf{Y}$ in order to detect the $N$ coded symbols $x_{j n}$ transmitted by the user $j$. Finally soft demapping and turbo decoding are carried out.

\section{COMBINING MC-CDMA AND SPATIAL MULTIPLEXING}

The MIMO MC-CDMA transmission and reception schemes are presented in Figure 1 and Figure 2 respectively.

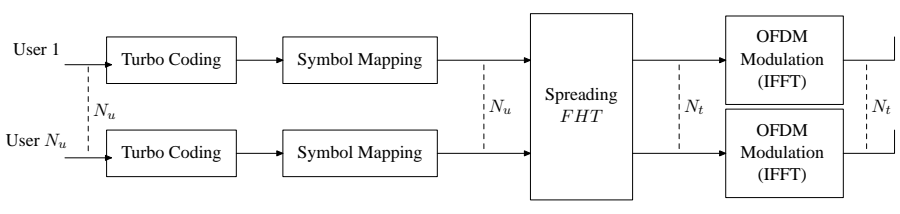

Fig. 1. MC-CDMA MIMO Transmitter

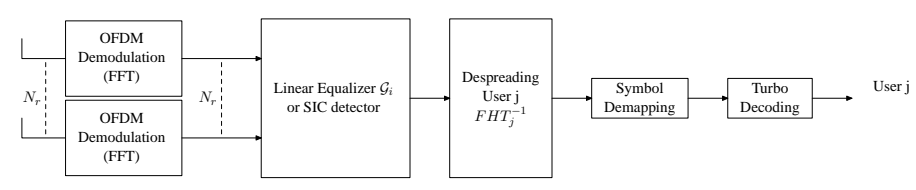

Fig. 2. MC-CDMA MIMO Transmitter and Receiver

\section{A. Transceiver}

First, data bit stream of each user is turbo coded individually and converted into a symbol vector. The multiuser turbo coded symbol matrix $\mathbf{X}$ including the information of all the users is send over $N_{t}$ transmit antenna. Then, the multiuser coded sequence is spread using a FHT coded symbol as for classical MC-CDMA. The transmitted matrix $\mathcal{S}$ of size $N_{t} L_{c} \times N$ is therefore given by:

$$
\mathcal{S}=\mathcal{C} . \mathcal{X}
$$

where the spreading sequences are given by the following equation $\mathcal{C}=\mathbf{I}_{N_{t}} \otimes \mathbf{C}$ of size $N_{t} L_{c} \times N_{t} N_{u}$ and where $\mathcal{X}$ is the $N_{t} N_{u} \times N$ matrix of multiuser coded sequences. Uncorrelated frequency non-selective Rayleigh fading per subcarrier is assumed as well as perfect channel estimation. The theoretical channel response, for the $k^{\text {th }}$ subcarrier, from transmit antenna $t$ to receive antenna $r$ can be estimated by $h_{r t, k}=\rho_{r t, k} e^{i \theta_{r t, k}}$. In the MIMO case, considering that the columns of the received matrix $\mathcal{R}$ of size $N_{r} L_{c} \times N$ are given by the vector $\mathbf{r}_{i}$ of length $N_{r} L_{c} \times 1, \forall i \in[1 \ldots N]$ during $N$ adjacent OFDM symbols, we obtain the following matrix representation:

$$
\mathbf{r}_{i}=\mathcal{H}_{i} \cdot \mathbf{s}_{i}+\mathbf{n}_{i} \quad \forall i \in[1 \ldots N]
$$

where the AWGN vector $\mathbf{n}_{i}$ is a vector of length $N_{r} L_{c} \times 1$ and where the channel matrix $\mathcal{H}_{i}$ is a $N_{r} L_{c} \times N_{t} L_{c}$ time varying matrix

$$
\mathcal{H}_{i}=\left[\begin{array}{cccc}
\mathbf{H}_{i}^{11} & \mathbf{H}_{i}^{12} & \ldots & \mathbf{H}_{i}^{1 N_{t}} \\
\mathbf{H}_{i}^{21} & \mathbf{H}_{i}^{22} & \ldots & \mathbf{H}_{i}^{2 N_{t}} \\
\vdots & \vdots & \ddots & \vdots \\
\mathbf{H}_{i}^{N_{r} 1} & \mathbf{H}_{i}^{N_{r} 2} & \ldots & \mathbf{H}_{i}^{N_{r} N_{t}}
\end{array}\right]
$$


where $\mathbf{H}_{i}^{r t}$ is the $L_{c} \times L_{c}$ time varying diagonal matrix with $h_{i}^{r t, k}$ the $k^{t h}$ element at time i.

\section{B. Decoding and MC-CDMA Equalization}

As for SISO, in order to restore the orthogonality between users, an equalization process is done before despreading. This equalization process has to take into account the CoAntenna Interference (CAI) caused by the spatial multiplexing scheme. In this section, we present two types of MIMO MCCDMA detectors, which are the SU MMSE detector adapted to a spatial multiplexing transmission and the SU SIC-MMSE detector without ordering. The SIC is carried out only on the multi-antenna received symbols which have CAI and not for the multi-user detection which is a SU detection.

1) SU MMSE detector adapted to spatial multiplexing: The equalization process consists of applying an equalization matrix $\mathcal{G}_{i}$ to the received vector $\mathbf{r}_{i}$. If we choose a SU MMSE equalizer, the equalization matrix is :

$$
\mathcal{G}_{i}=\frac{\mathcal{H}_{i}^{H}}{\mathcal{H}_{i}^{H} \mathcal{H}_{i}+\frac{1}{\gamma} \mathcal{I}}
$$

where $\gamma$ is the Signal to Noise Ratio at the receive antenna. After the decoding process we have:

$$
\hat{\mathbf{s}}_{i}=\mathcal{G}_{i} \mathbf{r}_{i}=\mathcal{G}_{i} \mathcal{H}_{i} \mathbf{s}_{i}+\mathcal{G}_{i} \mathbf{n}_{i} \quad \forall i \in[1 \ldots N]
$$

In order to retrieve the information, a despreading on the different layers of the resulting symbols is needed. The layer $l$ corresponds to a signal transmitted by one of the $N_{t}$ transmit antenna. For the despreading, the estimated soft symbols of user $j$ are given by the following vector of length $N_{t} L_{c} \times 1$ :

$$
\hat{\mathbf{x}}_{j}^{l}=\mathcal{C}_{j}^{H} \hat{\mathbf{s}}^{l}
$$

with $\mathcal{C}_{j}^{H}$ the transconjugate of user $j$ 's matrix spreading code over $N_{t}$ transmit antennas of size $N_{t} \times N_{t} L_{c}$. This leads to the following detector:

\section{SU MMSE detector adapted to spatial multiplexing}

$$
\begin{aligned}
& \text { for } 1=1 \text { to } n_{T} \\
& \text { - for } \mathrm{i}=1 \text { to } N \\
& -\mathcal{G}_{i}=\mathcal{H}_{i}^{+} \\
& -\hat{\mathbf{s}}_{i}=\mathcal{G}_{i} \mathbf{r}_{i} \\
& \text { - end for } \\
& \quad-\hat{\mathbf{x}}_{j}^{l}=\mathcal{C}_{j}^{H} \hat{\mathbf{s}}^{l} \\
& \text { end for }
\end{aligned}
$$

where $[.]^{+}$is the Moore-Penrose pseudo-inverse operation. This SU MMSE detector adapted to spatial multiplexing is later called SU MMSE detector.
2) SU SIC-MMSE detector: However, one can take advantage of the detections of the different layers by a SIC detector as described in [5]. We use a SIC detector without ordering. The ordering policy is not implemented because it does not improve the performance of spatial multiplexed MC-CDMA without ordering. In fact, the optimum ordering policy cannot be applied to spatial multiplexed MC-CDMA because the SIC algorithm cancels spreaded symbols due to CAI interference while the decisions has to be made on despreaded symbols. Therefore, these despreaded symbols don't take advantage of the MIMO channel since the despreading operation is performed over $L_{c}$ spreaded symbols affected by different subchannels.

First, a despreading on the first layer of the resulting symbols is needed leading to the soft estimated symbols of the different users given by the following vector of length $N_{t} L_{c} \times 1$ :

$$
\hat{\mathbf{x}}^{1}=\mathcal{C}^{H} \hat{\mathbf{s}}^{1}
$$

with $\mathcal{C}^{H}$ the transconjugate of users matrix spreading code over $N_{t}$ transmit antennas of size $N_{t} \times N_{t} L_{c}$. It is possible to make a hard decision on the estimated symbols of the different users according to the chosen modulation $\tilde{\mathbf{x}}^{1}=Q\left(\hat{\mathbf{x}}^{1}\right)$. When the soft or hard decisions of the first layer for the different users are made, it is needed to estimate the spreading symbols by the following formula $\tilde{\mathbf{s}}^{1}=\mathcal{C} \tilde{\mathbf{x}}^{1}$. From the V-BLAST detector [5], the estimated symbols from the different users need to be withdrawn from the received vectors $\mathbf{r}_{i}$ :

$$
\mathbf{r}_{i} \leftarrow \mathbf{r}_{i}-\mathcal{D}_{i}^{1} \cdot \tilde{\mathbf{s}}_{i}^{1} \quad \forall i \in[1 \ldots N]
$$

with

$$
\mathcal{D}_{i}^{1}=\left[\begin{array}{llll}
\mathbf{H}_{i}^{11} & \mathbf{H}_{i}^{21} & \ldots & \mathbf{H}_{i}^{N_{r} 1}
\end{array}\right]^{T} \quad \forall i \in[1 \ldots L]
$$

The equivalent channel matrix $\mathcal{H}_{i}$ becomes:

$$
\mathcal{H}_{i}=\left[\begin{array}{ccc}
\mathbf{H}_{i}^{12} & \ldots & \mathbf{H}_{i}^{1 N_{t}} \\
\mathbf{H}_{i}^{22} & \ldots & \mathbf{H}_{i}^{2 N_{t}} \\
\vdots & \ddots & \vdots \\
\mathbf{H}_{i}^{N_{r} 2} & \ldots & \mathbf{H}_{i}^{N_{r} N_{t}}
\end{array}\right]
$$

with $\mathbf{H}_{i}^{r t}$ the diagonal matrix channel of size $L_{c} \times L_{c}$ between transmit antenna $t$ and receive antenna $r$ for the time $i$. Then a new equalization matrix $\mathbf{G}_{i}$ is calculated. The precedent step is carried out iteratively until all layers are detected. We summarize all these steps by the following detector for the different users: 


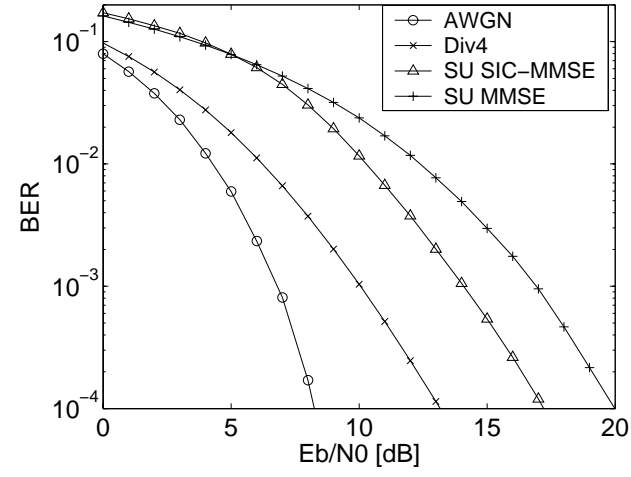

Fig. 3. Bit error rate performance of a $8 \mathrm{bps} / \mathrm{Hz}$ MIMO MC-CDMA system without channel coding

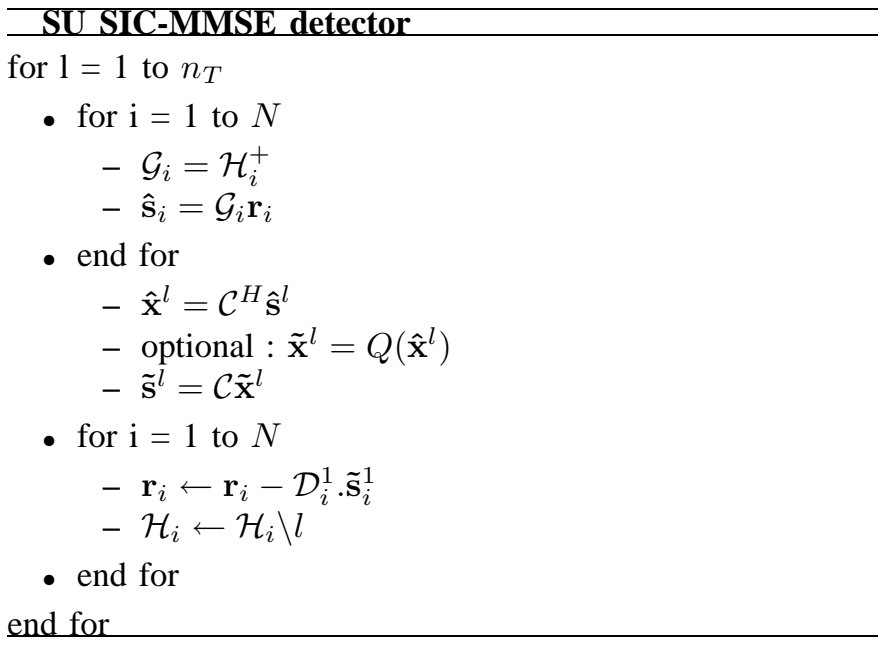

\section{Simulation Results}

In order to compare these two detectors, we carried out simulations in uncorrelated Rayleigh flat fading channels subcarriers first for uncorrelated antennas, then for different correlation between antennas. We use QPSK modulation with $N_{t}=4$ and $N_{r}=4$ leading to a $8 \mathrm{bps} / \mathrm{Hz}$ system without channel coding. We compare simulations for different load cases. For full load system, the number of active users $N_{u}$ is equal to the length of the spreading code $L_{c}=64$ and the number of subcarriers $N_{c}=64$.

Figure 3 shows the BER performance of the SU MMSE detector and the SU SIC-MMSE detector without channel coding at full load. Figure 4 shows the performance of these two detectors at a BER $10^{-3}$ for different loads. The curve

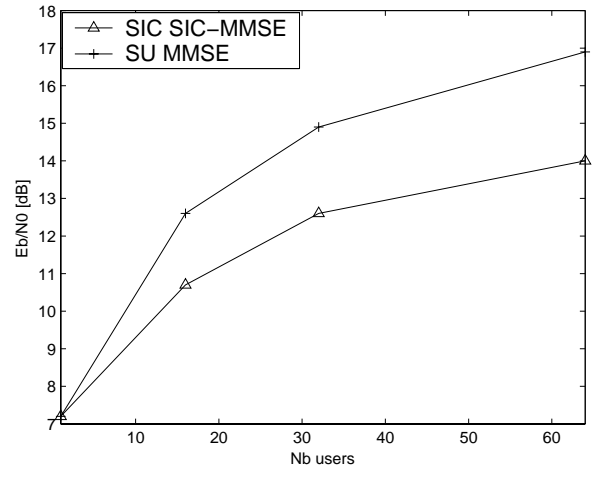

Fig. 4. Required $\mathrm{Eb} / \mathrm{NO}$ for a BER of $10^{-3}$ of a $8 \mathrm{bps} / \mathrm{Hz}$ MIMO MCCDMA system without channel coding

Div4 is the theoretical curve of a system with four antennas leading to a 4 diversity order. One can see that the full load curve is parallel to the theoretical curve due to the same exploited diversity order. The MIMO capacity is expressed by:

$$
C=n \log _{2}\left(1+\frac{\gamma}{N_{t}} \chi_{2 m}^{2}\right)
$$

with $n=\min \left(N_{t}, N_{r}\right)$ and $m=\max \left(N_{t}, N_{r}\right)$ and $\gamma$ the SNR at the receive antenna. For the full load curve in the $4 \times 4$ system, $n=m=4$ therefore full capacity and diversity are reached. In the lower load cases, the performance curve is obviously better due to less Multi Access Interference (MAI). We observe that the performance of the SU SICMMSE detector is better than the SU MMSE detector. This can be explained by the degrees of freedom introduced by the SIC detector which reduces the noise plus CAI and then increases the reliability of the decisions.

Figure 5 shows the BER performance of the same systems including a duo-binary Convolutional Turbo Code of rate $\frac{1}{2}$ at full load [6]. The encoding turbo code block size is equal to 432 (54 bytes) [7] and we carry out 6 iterations of turbo decoding. Figure 6 shows the performance of these two detectors at a BER $10^{-3}$ for different loads. Thus, we compare the systems at spectral efficiencies of $\eta=4 \mathrm{bps} / \mathrm{Hz}$ for both detectors. We observe that the performance of the SU SICMMSE detector leads to worse results than the SU MMSE one. In fact, compared to the SU MMSE detector that doesn't take any decisions the SU SIC-MMSE one can provide bad decisions harmful for channel decoding. Moreover, the BER performance with TC mainly depends on performance at low SNR before decoding, slightly better for SU MMSE detector. Therefore, the combination of a SU MMSE detector for a 


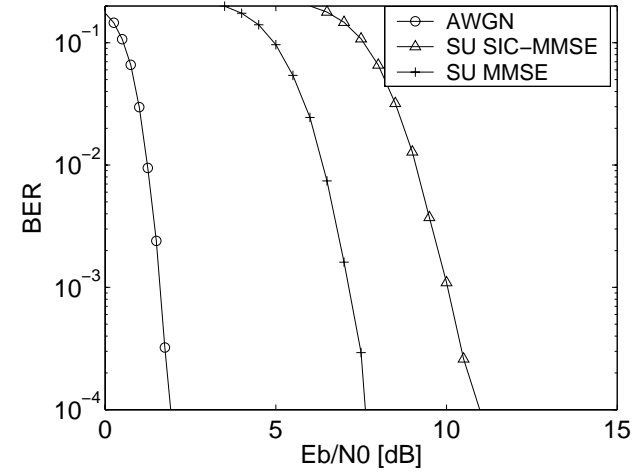

Fig. 5. Bit error rate performance of a $4 \mathrm{bps} / \mathrm{Hz}$ turbo coded MIMO MCCDMA system with channel coding

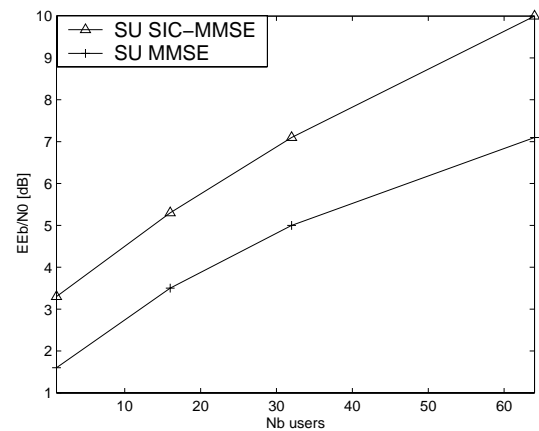

Fig. 6. Required Eb/N0 for a BER of $10^{-3}$ of a $4 \mathrm{bps} / \mathrm{Hz}$ turbo coded MIMO MC-CDMA system with channel coding

turbo coded MC-CDMA system is a low complexity efficient solution without using any SIC detectors to remove CAI.

Figure 7 shows the influence of the correlation between antennas for the SU MMSE detector with and without channel coding. We compare this detector without correlation and with a correlation matrix of $20 \%$ over the adjacent antennas, considering that the correlation is negligible between other antennas [8]. The chosen correlation matrix is:

$\left[\begin{array}{llll}1.0 & 0.2 & 0.0 & 0.0 \\ 0.2 & 1.0 & 0.2 & 0.0 \\ 0.0 & 0.2 & 1.0 & 0.2 \\ 0.0 & 0.0 & 0.2 & 1.0\end{array}\right]$

We observe that the performance loss between correlated and non correlated curves diminishes when channel coding is used, i.e $0.7 \mathrm{~dB}$ with channel coding instead of $3 \mathrm{~dB}$ at BER $10^{-4}$ owing to the BER before decoding as previously mentioned.

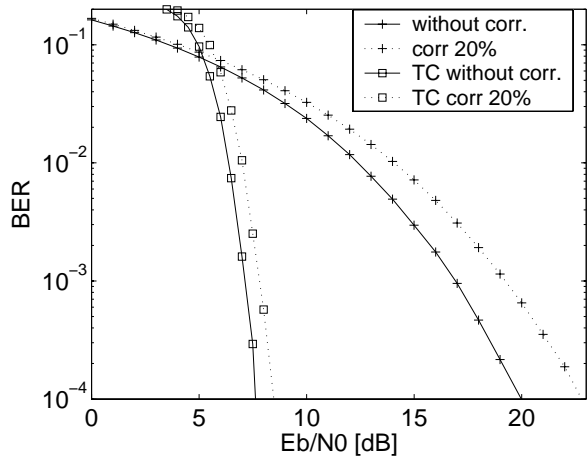

Fig. 7. Influence of the correlation on the SU MMSE detector with and without channel coding

\section{CONCLUSION}

In this paper, we have first extended SISO MC-CDMA to MIMO MC-CDMA systems. We have tested two types of multi-antenna receivers, both based on SU MMSE detector and the second one including multi-antenna SIC detection. Without channel coding, the SU SIC-MMSE detector gives the best results compared to the SU MMSE detector. However, with channel coding, the SU MMSE detector gives better results than the SU SIC-MMSE detector. Therefore, the combination of a SU MMSE detector for a turbo coded MIMO MC-CDMA system is a low complexity efficient solution without using any SIC detectors. Future work will focus on systems including more realistic MIMO channels.

\section{REFERENCES}

[1] N. Yee, J. P. Linnartz, G. Fettweis, Multicarrier CDMA in Indoor Wireless Radio Networks, IEEE PIMRC'93, pp. 109-113, Yokohama, Japan, 1993.

[2] G. J. Foschini, Layered space-time architecture for wireless communication in a fading environment when using multi-element antennas, Bell Labs Technical Journal, Vol. 1, No. 2, 1996, pp. 41-59.

[3] M. Hélard, R. Le Gouable, J.-F. Hélard, J.-Y Baudais, Multicarrier CDMA Techniques for Future Wideband Wireless Networks, Annales des Telecoms, Special issue on UMTS, May 2001.

[4] V. Le Nir, M. Hélard, R. Legouable Space-Time Block Coding Applied to Turbo Coded Multicarrier CDMA, Vehicular Technology Conference, Jeju, South Korea, 22-25 April 2003.

[5] P. W. Wolniansky, G. J. Foschini, G. D. Golden, R. A. Valenzuela V-BLAST: An architecture for realizing very high data rates over the rich-scattering wireless channel, Proc. IEEE ISSSE-98, Pisa, Italy, 30 September 1998

[6] C. Berrou, M. Jézéquel, Non binary convolutional codes for turbo-coding, Electronic Letters, vol. 35, N. 1, January 1999, pp. 39-45.

[7] ETSI EN 301958 V1.1.1 Digital Video Broadcasting (DVB); Interaction channel for Digital Terrestrial Television (RCT) incorporating Multiple Access OFDM, march 2002.

[8] S. Loyka Channel Capacity of MIMO Architecture Using the Exponential Correlation Matrix IEEE Communication Letters, Vol. 5, No. 9, September 2001 